\title{
La evolución en el aprendizaje del alumnado a través de la contrapráctica en la asignatura de Derecho Eclesiástico del Estado
}

\section{Núria Reguart Segarra ${ }^{a}$ y Victoria Camarero Suárez ${ }^{b}$}

a Doctora por la Universitat Jaume I, reguart@uji.es y ${ }^{\mathrm{b}}$ Profesora Titular de Derecho Eclesiástico del Estado de la Universitat Jaume I, csuarez@uji.es.

\begin{abstract}
In view of the growing need to reinforce the students' learning process, the counterpractice is projected as an innovative educational tool capable of achieving a learning evolution. Through this activity, which has already been implemented in the subject of State Ecclesiastical Law at the Jaume I University of Castellón, the student is granted the possibility of studying in depth a highly topical issue to prepare a legal reflection. Their previous autonomous work will be complemented and perfected with a rigorously updated legal-practical exposition by the teacher after which she will propose to the student an experimental practice with the aim of contrasting it with the legal reality. The learning evolution will take place when students are able to carry out an analysis of factual and legal basis, but enriched with the empirical contrast based on personal or third-party experiences that can lead to a major debate in relation to possible mismatches between law and social reality.
\end{abstract}

Keywords: counterpractice, learning evolution, autonomous work, legal-practical exposition, experimental practice, comparison.

\section{Resumen}

Ante la creciente necesidad de reforzar el proceso de aprendizaje del alumnado, la contrapráctica se proyecta como una herramienta educativa innovadora capaz de conseguir la evolución en dicho aprendizaje. A través de esta actividad, que ya se ha puesto en práctica en la asignatura de Derecho Eclesiástico del Estado por parte del profesorado de la Universitat Jaume I de Castellón, se otorga al alumno la posibilidad de estudiar a fondo un tema de gran actualidad para realizar una reflexión jurídica. Su trabajo autónomo previo se verá complementado y perfeccionado con una exposición jurídico-práctica rigurosamente actualizada por parte del docente tras la que este planteará al estudiantado una práctica experimental con el objetivo de contrastarla con la realidad jurídica. La evolución en el aprendizaje tendrá lugar cuando el alumnado sea capaz de llevar a cabo un análisis de fundamentación fáctico y jurídico, pero enriquecido con el contraste empírico basado en experiencias personales o de terceros que pueda conducir a un debate de gran calado en relación a posibles desajustes entre el derecho y la realidad social.

Palabras clave: contrapráctica, evolución en el aprendizaje, trabajo autónomo, exposición jurídico-práctica, práctica experimental, contraste. 


\section{Introducción}

El proceso de aprendizaje del alumnado se erige, en la actualidad, como el centro neurálgico alrededor del que se estructura el sistema educativo vigente (Reguart Segarra y Camarero Suárez, 2017). En consecuencia, cada vez es más frecuente encontrarse con toda una serie de actividades que deben ir desarrollándose a lo largo del curso académico para poder comprobar que efectivamente el estudiantado está asimilando los conceptos al ritmo que de ellos se espera. No obstante, en el caso del Grado en Derecho, esta tarea resulta particularmente dificultosa para el docente debido a la complejidad intrínseca de las materias jurídicas que deben someterse a evaluación, en las que, en la gran mayoría de casos, no hay respuestas erróneas o correctas per se, sino que cobra especial relevancia la argumentación jurídica aducida por el estudiante (Reguart-Segarra, Marullo, Camarero-Suárez, Zamora-Cabot y Carceller-Stella, 2019). En demasiadas ocasiones, los alumnos recurren a lo dispuesto en instrumentos legales, bien citándolos textualmente o empleando la técnica del parafraseo, sin que de ello pueda extraer el profesorado una conclusión certera acerca de si verdaderamente el alumno sabe de lo que está hablando. A la vista de esta problemática, desde el Área de Derecho Eclesiástico del Estado se ha impulsado una actividad novedosa que ya ha generado óptimos resultados: la contrapráctica.

Esta actividad se sitúa a caballo entre la tradicional clase teórica basada en la lección magistral, predominante en el ámbito jurídico, y la clase práctica en la que el protagonista es el alumno. Se combinan ambas modalidades de enseñanza debido a que se ha advertido que, con frecuencia, el alumno asume un rol eminentemente pasivo en las lecciones magistrales, en las que suele limitarse a escuchar lo que el docente ha de transmitirle. Por ello, tan solo se muestra capaz de desempeñar un rol activo en las clases prácticas, que, desafortunadamente, son más bien escasas en el Grado en Derecho. A diferencia de lo anterior, en la contrapráctica, el rol del alumno, así como el del docente, van cambiando a lo largo de su desarrollo para adecuarse a las necesidades de cada una de las cuatro fases en que se estructura y que más tarde se expondrán. Esta innovación se proyecta sobre el modo en que el estudiantado adquiere sus conocimientos en dos momentos distintos (uno previo a la lección magistral y otro posterior) y los pone en práctica ante un supuesto de gran actualidad, de forma que lo que se aspira a fomentar es la evolución en el aprendizaje, pero sin apartarse para ello de la tradicional lección magistral, que sigue siendo la modalidad organizativa por excelencia en este ámbito.

La actividad que en esta comunicación se presenta se ha puesto en práctica en los dos grupos de la asignatura de Derecho Eclesiástico del Estado que cursa el alumnado de primera anualidad del Grado en Derecho de la Universitat Jaume I de Castellón en cuanto Ciencia Jurídica Básica (referencia: DR1006). Como se ha indicado, consta de dos grupos (A y B) con un número bastante elevado de matrícula, que suele oscilar, en total, entre los 150 y 180 alumnos. Asimismo, se ha puesto en funcionamiento en el grupo del primer curso del Doble Grado en Administración de Empresas y Derecho (referencia: DA0106), recientemente implantado en la Universitat Jaume I, en el que cursan esta asignatura un máximo de 10 alumnos, lo que permite una observación del desarrollo de esta actividad mucho más cercana y minuciosa. Cabe señalar, también, que esta actividad se enmarca en el Plan de Formación y Mejora Educativa de Profesorado Novel correspondiente a la segunda anualidad (curso 2018/19) del Programa de Formación de Profesorado Novel de la Universitat Jaume I, en el que hemos participado en calidad de Profesora Novel (Núria Reguart) y Tutora de esta (Victoria Camarero).

\section{Objetivos}

El objetivo que se persigue radica, principalmente, en profundizar en la evolución progresiva del aprendizaje del alumnado, para lo cual resulta imprescindible tanto su trabajo autónomo previo como el 
que se deberá llevar a cabo el día previsto para la realización de la contrapráctica en el aula. Del mismo modo, se aspira a desarrollar la capacidad del estudiantado de fundamentar jurídicamente un tema de actualidad en el ámbito de la asignatura que reviste gran complejidad jurídica y para lo cual, con frecuencia, un alumno de primer año no está todavía preparado. Por ello, y a la vista de los graves problemas que suele acarrear el aplazamiento del desarrollo de esta capacidad primordial para un jurista a cursos posteriores, se ha considerado imprescindible que el alumnado se inicie en ella ya en su primera anualidad.

Así, esta modalidad de enseñanza pretende desarrollar el pensamiento crítico, el razonamiento y la capacidad de argumentación jurídica del alumnado, pero lo va a tomar en consideración en dos momentos distintos, como seguidamente se explicará. Ante todo, contribuye muy positivamente a la formación jurídica del estudiantado de primer curso al resultar de gran utilidad en la configuración de una amplia capacidad de asimilación de la problemática sociojurídica desde distintas perspectivas.

\section{Desarrollo de la innovación}

\subsection{Metodología}

Como ya se ha señalado, las modalidades organizativas en que se basa esta actividad son la clase teórica y la clase práctica, pero, además, se va a prestar especial atención a un momento previo, de manera que el trabajo autónomo realizado por el estudiantado con anterioridad al desarrollo presencial de esta actividad en el aula universitaria va a resultar crucial para la obtención de los resultados. Así, los métodos de enseñanza-aprendizaje que se combinan son el método expositivo, el estudio de casos (Limpias, 2012; Pérez Fuentes, 2016; Fernández-Izquierdo et al., 2018) y la resolución de problemas.

Con al menos un mes de antelación a la fecha fijada para el desarrollo de la actividad, se subirá a la plataforma virtual Moodle una tarea práctica a realizar individualmente por cada alumno. En el caso que nos ocupa, nos hemos decantado por una reflexión jurídica acerca de la problemática del uso del velo integral en los espacios públicos europeos. En las instrucciones para la realización de la práctica, tan solo se va a indicar que este asunto deberá valorarse a la luz del principio de libertad religiosa que define la esencia e identidad del Estado español; de la seguridad pública como elemento constitutivo del orden público; de la autonomía de la voluntad de las ciudadanas que deciden libremente portar el velo integral; de los principios de igualdad de sexos y dignidad de la mujer; así como de la protección de los derechos y libertades de los demás en cuanto límite legítimo al ejercicio del derecho de libertad religiosa. A partir de ahí, se insta a los alumnos a que desarrollen una reflexión jurídica acerca de la temática, cogiendo, para ello, como punto de partida algunos libros de referencia (Camarero Suárez, 2012; Motilla, 2009; Amérigo y Pelayo, 2013).

La sesión en la que se efectuará la contrapráctica quedará organizada del modo expuesto en la tabla que a continuación se muestra:

Tabla 1. Organización de la sesión

\begin{tabular}{cccc}
\hline Tiempo & Actividad & Rol del docente & Rol del alumnado \\
\hline $40^{\prime}$ & Exposición oral & Activo & Pasivo \\
$10^{\prime}$ & Contrapráctica & Activo & Activo \\
$30^{\prime}$ & Reflexión individual & Pasivo & Activo \\
$40^{\prime}$ & Debate & Pasivo & Activo \\
\hline
\end{tabular}

(c) 2020, Universitat Politècnica de València

Congreso In-Red (2020) 


\subsubsection{Fase 1: exposición oral}

Tras el trabajo autónomo previo, ya en el día asignado para la realización de la contrapráctica, los alumnos deberán acudir a clase con la tarea práctica que ya habrán subido al Aula Virtual impresa o en sus respectivos ordenadores portátiles. La clase comenzará con una exposición a cargo del experto en la que se tratará de plantear la problemática estudiada por los alumnos de un modo en que a estos les pueda resultar comprensible. Así, se abordará, en primer lugar, la más próxima actualidad jurídica y jurisprudencial ante una cuestión que cada vez suscita mayor interés público. A pesar de que el uso del velo integral en España es muy minoritario, ello no obsta para que se le deba otorgar la importancia que requiere un tema como este, en que se pone en juego la capacidad de un Estado aconfesional para regular una de las manifestaciones más llamativas de pertenencia religiosa.

Ante la presencia de este símbolo religioso en algunas zonas de Cataluña, ciertos Ayuntamientos de municipios catalanes decidieron acordar la prohibición del velo integral en determinados espacios públicos. En concreto, llegó al Tribunal Supremo el acuerdo del Pleno del Ayuntamiento de Lleida de 8 de octubre de 2010, lo que dio lugar al primer posicionamiento de este foro decisorio de primer orden acerca del velo integral en cuanto manifestación del derecho de libertad religiosa (Sentencia del Tribunal Supremo núm. 693/2013, de 14 de febrero, ECLI:ES:TS:2013:693). El centro neurálgico del caso gira en torno a la posible vulneración del artículo 16 de la Constitución española, que garantiza el derecho a la libertad ideológica, religiosa y de culto de los individuos y las comunidades sin más limitación, en sus manifestaciones, que la necesaria para el mantenimiento del orden público protegido por la ley. En el análisis de esta problemática, se debe partir de que la mujer es libre en su decisión de llevar el velo integral y que, por tanto, nos hallamos en el ámbito de la autonomía de la voluntad de las personas, que están facultadas para decidir libremente ejercer sus derechos fundamentales. En el presente caso, se había limitado de modo radical por medio de ordenanza municipal un derecho fundamental como lo es el de libertad religiosa. Sin embargo, el ejercicio del derecho de libertad religiosa, en cuanto derecho fundamental, tan solo puede ser limitado por medio de ley, de manera que unas ordenanzas municipales no pueden regular más que aspectos accesorios de los derechos fundamentales.

Asimismo, el Tribunal Supremo entró a valorar la alegada perturbación de la tranquilidad que implica la presencia del velo integral en nuestra sociedad, señalando que esta observación constituía un juicio de valor subjetivo en base al cual nunca podría justificarse el establecimiento de una prohibición general por parte de los poderes públicos. Por último, el Tribunal considera que los principios de igualdad de sexos y dignidad de la mujer no pueden, conjuntamente o de manera autónoma, constituir base suficiente para amparar una prohibición general de este símbolo religioso, puesto que no debe partirse del hecho de que la mujer no sea libre en la práctica del velo integral. Por todo lo anterior, el Tribunal concluye que la prohibición general del velo integral adolece de una clara fragilidad jurídica, por cuanto la limitación del ejercicio de este derecho fundamental tan solo cabe cuando sea absolutamente necesaria para la protección del orden público y de los derechos y libertades fundamentales de los demás.

Una vez estudiado el estado de la cuestión en el ordenamiento jurídico interno, se expondrá el primer pronunciamiento del Tribunal Europeo de Derechos Humanos (TEDH) sobre la materia, que tuvo lugar en 2014, en el caso S.A.S. contra Francia (Sentencia del TEDH en el caso S.A.S. c. Francia, de 1 de julio de 2014 [ap. núm. 43835/11]). S.A.S. son las siglas del nombre de una ciudadana francesa musulmana, nacida en 1990, que lleva el velo integral como consecuencia de su fe y de sus convicciones, por una decisión propia y sin ninguna voluntad de molestar a nadie. La aprobación de la Ley francesa núm. 20101191, de 11 de octubre, por la que se prohíbe el enmascaramiento del rostro en los espacios públicos va a tener una incidencia directa en el ejercicio de su derecho de libertad religiosa $\mathrm{y}$, en consecuencia, decide 
impugnarla. Llegado el asunto al TEDH, este enfoca su pronunciamiento en base a la violación de los artículos 8 y 9 del Convenio Europeo de Derechos Humanos (CEDH).

El TEDH analiza tres órdenes de argumentos en una línea bastante similar a la seguida por el Tribunal Supremo español. Sin embargo, cuando entra a valorar la protección de los derechos y libertades fundamentales de los demás en cuanto límite legítimo al ejercicio del derecho de libertad religiosa de los ciudadanos, llega a una conclusión radicalmente distinta. $\mathrm{Y}$ es que inserta en la protección de los derechos de los otros lo que se conoce como vivre ensemble, esto es, el respeto del conjunto mínimo de valores de una sociedad democrática y abierta, entendido como el respeto de las exigencias mínimas de la vida en sociedad. Comprende que las autoridades francesas han dado prioridad a este elemento por encima del derecho de las mujeres musulmanas a portar el velo integral por cuanto este símbolo religioso dificulta gravemente la interacción social, de manera que el TEDH acepta que un Estado considere esencial la interacción y comunicación de los individuos en una sociedad democrática, la cual puede verse alterada por el hecho de que algunos enmascaren su rostro en el espacio público. En consecuencia, y reconociendo que Francia gozaba en este caso de un amplio margen de apreciación por la falta de consenso europeo en la materia, concluye que no se han violado los artículos 8 y 9 del CEDH.

Como resulta deducible, esta decisión dio lugar a numerosas críticas y la doctrina no se ha mostrado uniforme acerca de si, en efecto, el velo integral dificulta la interacción social y debe ser prohibido, o si más bien constituye una manifestación esencial del derecho de libertad religiosa que tan solo debe limitarse por estrictas razones de orden público, cuando se haya probado un peligro cierto y constatado. El TEDH, no obstante, parece haber ratificado esta decisión en pronunciamientos posteriores (Sentencia del TEDH en el asunto Belcacemi y Oussar c. Bélgica [Sección 2ª], de 11 de julio de 2017 [ap. núm. 37798/13]; Sentencia del TEDH en el asunto Dakir c. Bélgica [Sección 2a], de 11 de julio de 2017 [ap. núm. 4619/12]) a propósito de leyes similares que se han promulgado en otros países europeos.

A través de esta exposición complementaria para el alumnado, se pretende facilitar información, promover la comprensión de conocimientos y estimular su motivación (De Miguel Díaz, 2006, 2005a y 2005b). Ello se debe a que, a pesar de que se presente un tema ya iniciado por el estudiantado, su dificultad jurídica puede provocar que el análisis autónomo de esta problemática por parte de estudiantes de primer curso no resulte del todo fructífero, puesto que se tratan conceptos que suelen ser todavía desconocidos para ellos. Así, la explicación desarrollada jurisprudencialmente por parte del docente debe estar enfocada a promover la comprensión y evolución de tales conceptos, por lo que el lenguaje a utilizar deberá ser claro y carecer de ambigüedades. Con tal fin, el docente deberá seleccionar, como estrategias metodológicas a utilizar en el desarrollo de su exposición, las siguientes:

- Realizar una buena introducción del tema para contextualizarlo y despertar el interés del alumnado, acompañada del guion que va a seguirse en la sesión.

- Prestar especial atención a la claridad, expresividad y ritmo de la exposición, para lo cual son determinantes las pausas y nexos, así como el énfasis en ciertos conceptos y la realización de resúmenes orales cada corto espacio de tiempo.

- Llevar a cabo una actualización jurídica y jurisprudencial al máximo nivel tomando en consideración que esta labor no puede ser exigida al conocimiento del alumnado en el momento de la sesión, por sobrepasar en gran manera el esfuerzo requerido.

- Evitar la formulación de preguntas al estudiantado en esta primera fase, pues su rol se ciñe a asimilar lo que el docente tiene que transmitirles para acometer, a continuación y utilizando el método comparativo de contraste, la resolución del conflicto que se le plantea. El debate deberá dejarse para el final de la sesión, tras haber escuchado al docente, haber planteado dos situaciones experimentales y haber reflexionado individualmente. 


\subsubsection{Fase 2: contrapráctica}

En esta segunda fase va a desarrollarse la contrapráctica propiamente dicha, en la que tanto el profesorado como el alumnado van a tener un papel eminentemente activo. Va a consistir en la formulación de dos experiencias personales o de terceros por parte del docente en relación a la exposición que se acaba de realizar. La conclusión a la que llega el TEDH es que el enmascaramiento del rostro rompe la interacción social, la comunicación entre la ciudadanía. Ello implica que el uso del velo integral, en cuanto forma de enmascarar el rostro, va en contra del vivre ensemble, de las exigencias mínimas de la vida en sociedad. A partir de esta conclusión, la práctica experimental y contrastada pretende conseguir que el estudiantado reflexione acerca de si es cierto que el uso del velo integral restringe la interacción entre las personas. En esta contrapráctica, las experiencias vitales que el docente plantea, y en las que el alumnado se debe implicar, son las siguientes:

A) El docente se halla en un aeropuerto a la espera de que se le comunique la puerta de embarque de su vuelo. En este escenario, se encuentra una mujer que porta un nicab. Ella lo observa con lo más profundo de su rostro: sus ojos. Se acerca a él y le pregunta con claridad si puede indicarle cuál es la puerta de embarque que le corresponde a su vuelo. El docente se percata de que se trata de la misma puerta de embarque a la que él se dirige, así que le ofrece caminar juntos hacia ella. En ese breve lapso de tiempo, comparten una agradable conversación.

B) En esta segunda situación, es el docente quien necesita ayuda, pues es él quien se encuentra perdido. Avanza hacia él una mujer con sus cascos de música a todo volumen, absorta en su propio mundo y con sus ojos fijos en la pantalla de su smartphone. Con ello, entiende que esa mujer está transmitiendo a la sociedad que desea permanecer al margen de los problemas de los demás, por lo que desiste en preguntarle. Ella está en su derecho a ser una outsider. La Ley, afortunadamente, no se lo prohíbe, pero sí en Francia, con el aval del TEDH, a la mujer que porta el nicab.

Así, mediante el planteamiento de estas experiencias, se pretende que el alumnado reflexione y se posicione acerca de si es verídico que el velo integral per se impide la interacción social o si se ha brindado la oportunidad de poner en práctica la tolerancia y de realizar un ejercicio de empatía, situándose en el lugar del diferente en un modelo de integración que no debería ser entorpecido, por ejemplo, con una ley de prohibición general, que no es sino un golpe de autoridad desproporcionado e innecesario.

\subsubsection{Fase 3: reflexión individual}

En esta tercera fase, es el alumnado quien va a asumir el rol protagonista. Una vez realizada la exposición de la problemática por parte de un experto en ella, como lo es el docente de la asignatura de Derecho Eclesiástico del Estado, y planteadas estas dos experiencias en claro contraste entre ellas, el estudiantado deberá volver a analizar los mismos fundamentos de hecho y de derecho que ya había estudiado previamente a la sesión, pero en esta fase ya con un bagaje sociojurídico a un nivel considerable, para llegar a una decisión definitiva a favor o en contra de la problemática formulada. Deberá decidir, en base a los razonamientos jurídicos esgrimidos tanto por el Tribunal Supremo español como por el TEDH, así como a tenor de lo dispuesto en las diferentes leyes promulgadas en el contexto europeo, si, en efecto, es legítima la prohibición del uso generalizado del velo integral en los espacios públicos europeos. La conclusión final a la que llegue el alumnado en esta tercera fase no tiene por qué coincidir con la primera a la que llegó, que será la que figurará en la tarea subida al Aula Virtual con anterioridad al inicio de la sesión programada. Así, se hará saber que tanto la tarea colgada online como la reflexión jurídica final a la que lleguen en clase, que deberán plasmar por escrito y entregar al profesorado, van a tomarse en consideración a la hora de calificar la tarea en su evaluación continuada. 


\subsubsection{Fase 4: debate}

En la última fase de esta actividad, se abrirá el debate a toda la clase, para que pongan en común su acuerdo o desacuerdo con la realidad contrastada. Ellos mismos podrán darse cuenta de si la mayoría ha evolucionado en el aprendizaje gracias a la contrapráctica o si siguen estancados en un pensamiento que no les ha permitido avanzar. En esta fase, el profesor asumirá el rol de moderador del debate, de modo que otorgará los turnos de palabra, evitará que no se escuchen entre ellos y, por encima de todo, impedirá que los alumnos y alumnas abandonen los argumentos estrictamente jurídicos para esgrimir meras opiniones personales alejadas del ámbito jurídico-práctico. Por su parte, el alumnado deberá participar activamente en el debate y en las reflexiones que puedan originarse. Así, deberá dejar a un lado sus miedos e inseguridades para ser capaz de hablar en frente de sus compañeros defendiendo su propia postura sin que ello le suponga un excesivo esfuerzo emocional.

\subsection{Competencias adquiridas}

Las competencias que se aspira a que el estudiantado adquiera giran en torno a la asimilación de la incidencia y problemática jurídica del derecho de libertad religiosa y sus manifestaciones en la esfera pública. En cuanto competencias específicas, cabe destacar:

- Manejo y uso de las fuentes del Derecho eclesiástico del Estado.

- Consulta de jurisprudencia y doctrina relevante para el objeto de estudio.

- Mayor comprensión del derecho de libertad religiosa como primer derecho humano, radical e íntimo de la persona, cuyo ejercicio tan solo puede limitarse en situaciones tasadas.

- Análisis de la problemática planteada desde una perspectiva de estudio jurídico-sociológico y a través de argumentos jurídicos diferentes e, incluso, en ocasiones opuestos entre sí.

- Reflexión jurídica a favor o en contra de la prohibición general del velo integral en el espacio público a la vista de todo lo anterior.

- Evolución en la reflexión jurídica inicial tras la realización de la contrapráctica.

\section{Resultados}

En el curso académico 2018/19, un total de 160 alumnos participaron en la realización de la contrapráctica, de los cuales hasta 135 demostraron haber evolucionado positivamente en su aprendizaje, de manera que su conclusión final tras la realización de la contrapráctica progresó hacia un mayor entendimiento de la problemática jurídica que rodea a la prohibición del velo integral en los espacios públicos europeos. Veintiún alumnos continuaron en su pensamiento inicial, aunque cabe señalar que, en la mayoría de los casos, su pensamiento previo a la realización de la contrapráctica ya mostraba una gran capacidad de comprensión y razonamiento jurídico, por lo que la continuidad es también de signo positivo. Tan solo cuatro alumnos mostraron una evolución negativa, es decir, que tras la realización de la contrapráctica se reafirmaron inmotivadamente en un pensamiento muy básico y limitado que se alejaba de las líneas jurisprudenciales y doctrinales más actuales y que, por tanto, no era congruente con los objetivos planteados, lo que demuestra que es un factor crucial no solo atender detenidamente tanto a la exposición oral como a la contrapráctica en sí, sino también realizar un trabajo preparatorio previo satisfactorio.

El análisis de los datos obtenidos se ha realizado en base al siguiente razonamiento, que toma como punto de partida inicial la práctica subida al Aula Virtual por el estudiantado: 
- Evolución positiva. Se incluyen dentro de esta calificación todas aquellas reflexiones individuales escritas que, tras haber sido comparadas con la práctica inicial de cada alumno, demuestren que este ha comprendido los conceptos transmitidos por el docente en su exposición y que ha sido capaz de reflexionar sobre ellos y extraer una conclusión basada en la jurisprudencia y doctrina más actualizada. Se valorará, asimismo, positivamente la intervención en el debate final, si bien es cierto que, por motivos de tiempo y número de alumnado matriculado, no es factible que todos puedan participar en él.

- Evolución negativa. El alumnado cuya evolución se califica de negativa es aquel que ha aducido exactamente los mismos argumentos y fundamentación jurídica en la tarea colgada en el Aula Virtual y en la reflexión individual realizada tras la contrapráctica, o bien ha traído a colación unos argumentos nuevos que nada tienen que ver con lo explicado por el docente, sino que, por el contrario, los ha empleado en un modo opuesto al que el docente ha tratado de transmitirlos, sin justificación razonada alguna. Ello deja entrever que no ha adquirido las competencias esperadas y no ha sido capaz de seguir al docente en su exposición jurídico-práctica, por lo que su razonamiento crítico no se ha desarrollado conforme a lo esperado y se considera que se ha producido un estancamiento en su proceso de aprendizaje. Lo anterior suele ser consecuencia de una insuficiencia en el trabajo preparatorio previo y de una falta de motivación del estudiantado. La falta de preparación previa limita o, incluso, imposibilita la evolución en el aprendizaje, es decir, un alumno que no se haya preparado adecuadamente la práctica que debe subir al Aula Virtual no va a poder evolucionar en su aprendizaje, pues la asimilación de los conceptos transmitidos por el docente en la sesión en que se desarrolla la contrapráctica depende en gran medida del trabajo autónomo previo realizado por cada uno de los asistentes. Dicho de otro modo, la insuficiencia en el trabajo preparatorio previo explica la no asimilación por parte del alumno de los nuevos conceptos y, como resultado, su falta de evolución en el aprendizaje.

- Continuidad en el pensamiento. En este caso, si bien el alumnado ha seguido en su reflexión final la línea por él mismo expuesta en la tarea práctica subida al Aula Virtual, su trabajo autónomo plasmado en dicha tarea ya evidenciaba una gran labor de búsqueda jurisprudencial y doctrinal, una suma coherencia en su argumentación jurídica y en el desarrollo de su pensamiento crítico, y una clara exposición de la problemática en consonancia con lo anterior. Por ello, la reflexión individual no hace más que corroborar su pensamiento inicial, enriquecido con lo expuesto en clase por el docente y por la realización de la contrapráctica a través de la experiencia vital planteada.

Como puede apreciarse en las tablas que a continuación se muestran, el porcentaje de observación es bastante similar en los dos grupos en que se ha implementado esta actividad, y ello a pesar del abismo entre el número de matriculados en uno y otro, que obedece a motivos exclusivamente académicos relacionados con el plan de estudios de cada titulación. Asimismo, tras solicitarse al alumnado que valorara la actividad de forma anónima, prácticamente todos coincidieron en calificarla de altamente positiva para su formación, por cuanto se trata jurídicamente un tema de actualidad sobre el que no pocos se aventuran a opinar sin tener conocimientos técnicos al respecto.

Tabla 2. Resultados obtenidos en la asignatura DR1006

\begin{tabular}{c|cccc}
\cline { 2 - 4 } & $\begin{array}{c}\text { Tareas Aula } \\
\text { Virtual }\end{array}$ & Evolución positiva & Evolución negativa & $\begin{array}{c}\text { Continuidad en el } \\
\text { pensamiento }\end{array}$ \\
\hline $\begin{array}{c}\text { Número de } \\
\text { alumnado } \\
\begin{array}{c}\text { Porcentaje de } \\
\text { alumnado }\end{array}\end{array}$ & 151 & 128 & 3 & 20 \\
\hline
\end{tabular}


Tabla 3. Resultados obtenidos en la asignatura DA0106

\begin{tabular}{c|cccc}
\cline { 2 - 5 } & $\begin{array}{c}\text { Tareas Aula } \\
\text { Virtual }\end{array}$ & Evolución positiva & Evolución negativa & $\begin{array}{c}\text { Continuidad en el } \\
\text { pensamiento }\end{array}$ \\
\hline $\begin{array}{c}\text { Número de } \\
\text { alumnado }\end{array}$ & 9 & 7 & 1 & 1 \\
$\begin{array}{c}\text { Porcentaje de } \\
\text { alumnado }\end{array}$ & $100 \%$ & $77,8 \%$ & $11,1 \%$ & $11,1 \%$ \\
\hline
\end{tabular}

En los cursos siguientes, se deberá llevar a cabo un riguroso seguimiento de las variaciones que puedan darse en estos porcentajes. Con este fin, deberán compararse los resultados obtenidos en el curso académico 2018/19 con los que se obtengan en los cursos posteriores, lo que resultará crucial, a su vez, para poder comprobar la tasa de éxito global de superación de la asignatura antes y después de la introducción de la mejora educativa.

\section{Conclusiones}

Es bien sabido que el foco de atención principal sobre el que se articula el sistema educativo vigente radica en el aprendizaje del alumnado. Por ello, está en la mano de los docentes idear nuevas herramientas educativas y modalidades organizativas que favorezcan el aprendizaje y que sean de gran utilidad para desarrollar la plena capacidad de comprensión y asimilación de conceptos del estudiantado. Esta tarea se estima particularmente necesaria en el ámbito de las ciencias jurídicas, en las que con demasiada frecuencia se recurre a textos legales, doctrina y jurisprudencia que se escapan del nivel de asimilación del alumnado, generando resultados negativos por lo que se refiere a su proceso de aprendizaje.

Como ha quedado patente, la contrapráctica ha demostrado ser una actividad innovadora y eficaz para lograr su principal objetivo: la evolución en el aprendizaje. Una amplia mayoría del alumnado sometido a estadística consiguió evolucionar positivamente en su aprendizaje tras la realización de la contrapráctica y mostró un gran interés para su utilización en prácticas futuras, lo que arroja un resultado más que óptimo. Es por ese motivo por el que, desde el Área de Derecho Eclesiástico del Estado de la Universitat Jaume I, se seguirá apostando por esta herramienta educativa en los próximos cursos, siguiendo así las valoraciones del propio estudiantado, que la reputa de altamente beneficiosa para su formación como juristas.

\section{Referencias}

AMÉRIGO, F. y PELAYO, D. (2013). El uso de símbolos religiosos en el espacio público en el Estado laico español. Fundación Alternativas.

CAMARERO SUÁREZ, V. (2012). El velo integral y su respuesta jurídica en democracias avanzadas europeas. València: Tirant lo Blanch.

CORREA GOROSPE, J.M. (2005). "La integración de plataformas de e-learning en la docencia universitaria: Enseñanza, aprendizaje e investigación con Moodle en la formación inicial del profesorado" en Revista Latinoamericana de Tecnología Educativa, vol. 4, núm. 1, pp. 37-48.

DE MIGUEL DÍAZ, M. (2006). Metodologías de enseñanza y aprendizaje para el desarrollo de competencias. Madrid: Alianza Editorial. 
DE MIGUEL DÍAZ, M. (2005a). Modalidades de enseñanza centradas en el desarrollo de competencias. Oviedo: Universidad de Oviedo, Ministerio de Educación y Ciencia.

DE MIGUEL DÍAZ, M. (2005b). Adaptación de los planes de estudio al proceso de convergencia europea. Oviedo: Servicio de Publicaciones de la Universidad de Oviedo.

FERNÁNDEZ-IZQUIERDO, M.A., MUÑOZ-TORRES, M.J., RIVERA-LIRIO, J.M., FERRERO-FERRERO, I., ESCRIG-OLMEDO E. y MARULLO, M.C. (2018). "The 'case study' as a teaching tool for the integration of Sustainable Development Goals” en Proceedings of EDULEARN18 Conference, IATED, pp. 4036-4041.

HUERTA, R. (2016). "Formación de docentes y defensa de los derechos humanos mediante actividades educativas en museari.com" Botti Navarro, V. y Fernández Prada, M.A. (eds.). En Libro de Actas IN-RED 2016: II Congreso Nacional de Innovación Educativa y Docencia en Red. València: Editorial Universitat Politècnica de València.

LIMPIAS, J.L. (2012). "El método del estudio de casos como estrategia metodológica para desarrollar habilidades investigativas en la formación del jurista” en Revista Boliviana de Derecho, núm. 13, pp. 60-101.

LÓPEZ GÜETO, A. (2018). "El método del caso aplicado a la enseñanza del Derecho Romano" en Docencia y Derecho, Revista para la docencia jurídica universitaria, núm. 12, pp. 1-14.

LÓPEZ-SIDRO LÓPEZ, A. (2018). "Buenas prácticas en la docencia de la asignatura de Derecho y factor religioso: reflexiones desde la experiencia” en Revista General de Derecho Canónico y Derecho Eclesiástico del Estado, vol. 48 , pp. $1-25$.

MOTILlA, A. (coord.) (2009). El pañuelo islámico en Europa. Madrid: Marcial Pons.

ORDEÑANA GEZURAGA, I. (2018). "Los 'One minute paper' como elemento de evaluación continua en la búsqueda de la mejora de la evaluación en una asignatura del Grado en Derecho" Vega Carrero, V. y Vendrell Vidal, E. (eds.). En Libro de Actas IN-RED 2018: IV Congreso Nacional de Innovación Educativa y Docencia en Red. València: Editorial Universitat Politècnica de València (pp. 416-427).

PÉREZ FUENTES, G.M. (2016). "Estudio de casos y hechos como modalidad activa de la enseñanza del Derecho" en Actualidad Jurídica Iberoamericana, núm. 4 (bis), pp. 280-302.

PERIAGO MORANT, J.J. (2019). "TICS y Redes Sociales en derecho penal: Pensamiento analítico" Vega Carrero, V. y Vendrell Vidal, E. (eds.). En Libro de Actas IN-RED 2019: V Congreso Nacional de Innovación Educativa y Docencia en Red. València: Editorial Universitat Politècnica de València (pp. 488-501).

PONS-ESTEL TUGORES, C. y GONZÁLEZ SÁNCHEZ, M. (2018). "Materiales audiovisuales para el estudio de cuestiones de Derecho de Familia y Sucesiones a partir de la jurisprudencia de Estrasburgo" Vega Carrero, V. y Vendrell Vidal, E. (eds.). En Libro de Actas IN-RED 2018: IV Congreso Nacional de Innovación Educativa y Docencia en Red. València: Editorial Universitat Politècnica de València (pp. 220-229).

REGUART-SEGARRA, N., MARULlO, M.C., CAMARERO-SUÁREZ, V., ZAMORA-CABOT, F.J. y CARCELLER-STELLA, J.J. (2019). "Integrating the 'Business and Human Rights' discourse into the university classroom through collaborative learning” en Proceedings of INTED2019 Conference, IATED, pp. 1142-1148.

REGUART SEGARRA, N. y CAMARERO SUÁREZ, V. (2017). "Los seminarios como modalidades organizativas para generar la interacción y el debate entre el alumnado" en IV Jornada Nacional sobre Estudios Universitarios y II Taller de Innovación Educativa. Competencias: Formación y Evaluación. Castelló de la Plana: Publicacions de la Universitat Jaume I (pp. 417-424).

SÁNCHEZ BAYÓN, A. (2013). "El Derecho eclesiástico en las universidades estadounidenses: su estudio mediante jurisprudencia y estudios de casos” en Revista Española de Derecho Canónico, vol. 70, núm. 174, pp. $229-265$.

SUBERBIOLA GARBIZU, I. (2019a). "Claves para la creación entornos virtuales de aprendizaje de la asignatura Derecho Financiero I" Vega Carrero, V. y Vendrell Vidal, E. (eds.). En Libro de Actas IN-RED 2019: V Congreso Nacional de Innovación Educativa y Docencia en Red. València: Editorial Universitat Politècnica de València (pp. 11-25).

SUBERBIOLA GARBIZU, I. (2019b). "ODS en el Derecho Financiero y Tributario en la Universidad del País Vasco-Euskal Herriko Unibertsitatea" Vega Carrero, V. y Vendrell Vidal, E. (eds.). En Libro de Actas IN-RED 2019: $V$ Congreso Nacional de Innovación Educativa y Docencia en Red. València: Editorial Universitat Politècnica de València (pp. 1503-1513). 